\title{
Criminologie
}

\section{Comportements suicidaires et délinquance, des liens à (re)découvrir}

\section{Marc Daigle}

Volume 34, numéro 2, automne 2001

Comportements suicidaires et délinquance

URI : https://id.erudit.org/iderudit/027502ar

DOI : https://doi.org/10.7202/027502ar

Aller au sommaire du numéro

Éditeur(s)

Les Presses de l'Université de Montréal

ISSN

0316-0041 (imprimé)

1492-1367 (numérique)

Découvrir la revue

Citer ce document

Daigle, M. (2001). Comportements suicidaires et délinquance, des liens à (re)découvrir. Criminologie, 34(2), 3-7. https://doi.org/10.7202/027502ar d'utilisation que vous pouvez consulter en ligne.

https://apropos.erudit.org/fr/usagers/politique-dutilisation/ 


\section{Comportements suicidaires et délinquance, des liens à (re) découvrir}

Marc Daigle

Chercheur

Centre de recherche et d'intervention sur le suicide et l'euthanasie

Institut Philippe Pinel de Montréal

Professeur

Département de psychologie

Université du Québec à Trois-Rivières

marc_daigle@uqtr.uquebec.ca

Ce numéro de la revue Criminologie traite de deux réalités qui se recoupent, mais qui ne se laissent pas facilement cerner : les comportements suicidaires et la délinquance. Le concept de " comportements suicidaires " lui-même réfere à un ensemble d'événements qu'on appellera suicides, tentatives de suicide, automutilations mais aussi, à la limite, idéations suicidaires. En parallèle, la mesure du problème se ferait avec une estimation de l'urgence suicidaire (à plus court terme) ou du risque suicidaire (à plus long terme). Sans trop complexifier le débat, disons ici que le plus difficile est probablement de distinguer tentatives de suicide et automutilations. On dira que les premiers comportements correspondent, eux, à une véritable intention de mourir (Favazza, 1996; Fulwiler et al.,1997, par exemple) mais ce n'est pas si simple (Smith et al., 1984). Les personnes qui font des tentatives de suicide, ou qui s'automutilent, ne sont pas toujours conscientes de ce qui les anime ou peuvent bien nier plus tard le sens de leur geste. L'automutilation, comme telle, serait donc vraiment à la limite des comportements "suicidaires", certains auteurs la considérant 
comme un comportement plus ou moins adaptatif, quoique pathologique (Strong, 1998 ; Favazza, 1999). Or, dans ce numéro, Sylvie Frigon nous interpelle justement là-dessus, même si elle ne se réfere pas nécessairement aux mêmes distinctions. Dans une perspective de sociologie du corps, elle nous rappelle que l'automutilation, du moins chez les femmes incarcérées, peut être justement l'expression d'une résistance à l'enfermement, d'une reprise de contrôle. Son article a notamment le mérite de nous amener à saisir cette réalité du point de vue des femmes concernées personnellement par le phénomène, une approche méthodologique à laquelle nous invitent aussi Joel Harvey et Alison Liebling dans un autre texte.

Pour ce qui est du concept de délinquance, qui nous intéresse ici en fonction des comportements suicidaires, il a été délibérément retenu pour rendre compte d'une réalité qui est de plus en plus évidente. La prévalence des comportements suicidaires, incluant les comportements automutilatoires, est élevée chez les détenus ou chez les jeunes en garde fermée, mais elle est probablement aussi élevée chez les délinquants qui vivent en milieu libre. Or, comment faut-il désigner ces personnes qui ont des caractéristiques bien particulières et qui, souvent, transitent plusieurs fois entre milieu libre et milieu fermé ? Nous avons choisi de retenir ici le terme de délinquants qui, avec une certaine imprécision, nous permet d'inclure, non seulement les délinquants adultes de la rue ou de la prison, mais aussi les jeunes des Centres jeunesse aux comportements perturbateurs ou qui ont des troubles de comportement sévères. Dans ce numéro, l'article de François Chagnon, Johanne Renaud et Lambert Farand situe d'ailleurs très bien cette perspective où l'on considère que certaines personnes ont des caractéristiques qui les rendront éventuellement plus suicidaires, notamment l'impulsivité. Toujours dans cette perspective où les délinquants ne sont pas moins suicidaires à l'intérieur qu'à l'extérieur des murs, l'article de Daigle et Côté en appelle à une prise en charge par la communauté au sens large. Cette dernière approche rejoint celle prônée dans les stratégies de prévention anglaises, tel que le soulignent Joel Harvey et Alison Liebling, où le " problème " du suicide ne doit pas être la responsabilité unique des cliniciens de la prison, qu'ils soient criminologues, psychologues, médecins...

Harvey et Liebling, tout comme Martine Herzog-Evans pour la France, nous présentent une approche de la situation carcérale qui est plus sociologique et plus qualitative. Leur jugement sur les progrès faits 
dans les prisons d'Angleterre et du Pays de Galles est cependant beaucoup plus positif. Les auteurs en appellent cependant à des correctifs bien différents. Martine Herzog-Evans, en effet, privilégie l'exploration des pistes juridiques pour imposer une certaine contrainte aux administrations pénitentiaires. Harvey et Liebling, malgré une approche qui se veut plus sociologique que psychologique, misent plus sur une amélioration des relations interpersonnelles dans les prisons, sans que cela ne soit nécessairement formalisé dans des textes de loi. Sans doute est-ce là le reflet, non seulement des formations académiques différentes des auteurs, mais aussi de l'évolution différente du dossier de la prévention du suicide dans les régimes carcéraux français et anglais.

Quant à la situation précise de la France, notons que Martine Herzog-Evans présente le point de vue d'une juriste sensible au respect des droits des détenus, domaine où se situe principalement sa compétence ${ }^{1}$. Mais elle présente aussi le point de vue du " public " (avec tout le flou que comporte cette notion), ou du moins des administrés, qui ne décodent pas toujours ce qui semble être parfois, vu de l'extérieur, un état de crise permanent dans les prisons. Elle renvoie d'ailleurs ellemême à la parution en France du livre de Véronique Vasseur qui illustre bien cette atmosphère sociale. Plus spécifiquement, Martine HerzogEvans interpelle le système carcéral en ce qu'il augmenterait considérablement les risques de suicide chez les délinquants (thèse quelque peu différente de celle que nous défendons personnellement, un peu à la manière de Claude Balier, $1988^{2}$ ). Sans faire explicitement cette distinction, elle invoque néanmoins deux types de contraintes qui accableraient le détenu : celles inhérentes à l'incarcération comme telle (isolement, solitude, peur, privation de liberté) et celles relatives à certains manquements ou abus des gestionnaires (non-respect des normes, non-application des mesures ou, pire, manque d'humanisme ou de respect). L'allégement des premières contraintes ferait appel

1. Rappelons ici qu'une telle approche est courante aux États-Unis et qu'elle a mené à des compensations financières importantes pour les familles des victimes. Au Canada, notamment au Québec, cette perspective polémique est peu développée.

2. "Il est évident que la qualité de la prison, tant par son environnement architectural qu'humain, joue un rôle dans la répétition ou la sédation des manifestations bruyantes, ainsi que dans l'importance de la régression et de la passivité. Mais il faut des conditions extrêmes pour affirmer qu'elle crée à elle seule de toutes pièces des troubles qui ne préexistaient pas, sous une forme ou sous une autre, avant l'incarcération » (Balier, $1988: 20$ ). 
effectivement à une réforme du système carcéral, sinon de la société tout entière, ce qui est un débat qui dépasse ici nos objectifs. Quant aux contraintes de l'autre type, elles devraient être levées en priorité lorsqu'elles sont bien identifiées. Ici, justement, on peut penser que la gestion carcérale pourrait être resserrée, que les procédures sont perfectibles, que les mauvais employés peuvent être tamenés à l'ordre. II n'empêche qu'une telle façon de voir les choses nous éloigne encore plus d'une culture de concertation, certes fort idéaliste en milieu carcéral, mais qui permettrait quand même la mise en œuvre de véritables stratégies efficaces de prévention du suicide.

Finalement, deux constats s'imposent. Tout d'abord, notre opinion sur la question n'est probablement pas fondée que sur la raison. Elle est influencée par l'expérience subjective que nous avons du milieu carcéral. Dans la majorité des cas, c'est-à-dire pour la majorité des citoyens, cette expérience est presque nulle et, par conséquent, souvent bien naïve (quoique, dans certaines communautés américaines, par exemple, un fort pourcentage de citoyens puisse avoir connu l'incarcération, ne serait-ce que par personne interposée). Dans les autres cas, les expériences carcérales sont souvent interprétées à partir d'une perspective bien particulière et manichéenne. Que l'on soit détenu ou ex-détenu, bien évidemment, mais aussi gestionnaire, intervenant ${ }^{3}$, défenseur des droits des détenus ou des employés, chercheur universitaire... il faut demeurer bien modeste dans l'invocation de notre objectivité.

L'autre constat, c'est qu'il ne faut pas trop chercher, finalement, à obtenir l'unanimité dans ce milieu. Sans verser dans le défaitisme, disons que les principaux " partenaires " (!) du système vont (et doivent) conserver leurs positions. Le gestionnaire restera surtout préoccupé par la bonne marche de l'ensemble d'un programme de prévention du suicide et craindra d'être accusé de non-respect des droits des individus. Le défenseur des détenus (ou des jeunes des Centres jeunesse, récemment au Québec) continuera de relever les écarts aux droits, sans trop s'occuper des contraintes du quotidien et des autres priorités institutionnelles. Et c'est bien ainsi ! Il ne faut pas se fier uniquement à la bonne volonté ou à la bonne foi des gestionnaires et des intervenants. Tout comme il ne faut pas, non plus, se fier uniquement à l'application du

3. Sans compter, ici, les oppositions entre différents types d'intervenants. 
droit pour faire fonctionner une institution au quotidien et, encore moins, pour soulager la souffrance psychologique des personnes qui ont de multiples problèmes ${ }^{4}$.

\section{Références}

Balier, C. 1988. Psychanalyse des comportements violents, Paris : PUF.

Favazza, A. 1996. Bodies Under Siege: Self-mutilation and Body Modification in Culture and Psychiatry, Baltimore : The Johns Hopkins University Press.

Favazza, A. R. 1999. "Self-mutilation ", Pp. 125-145 in The Harvard Medical School Guide to Suicide Assessmeni and Intervention, sous la direction de G. Douglas et M. D. Jacobs. San Francisco : Jossey-Bass.

Fulwiler, C., Forbes, C., Santagelo, S. L. et Folstein, M. 1997. « Selfmutilation and Suicide Attempt: Distinguishing Features in Prisoners " Journal of the American Academy of Psychiatry and the Law 25 (1) : 69-77.

GrenIER, G. 1999. Les monstres, les fous et les autres. La folie criminelle au Québec, Montréal : Éditions Trait d'union.

LitMAN, R. E. 1980. "Psycholegal Aspects of Suicide", Pp. 840-853 in Modern Legal Medicine, Psychiatry, and Forensic Science, sous la direction de W. J. Curran, A. L. McGarry et C. S. Petty. Philadelphia : Davis.

SMITH, K., CONROY, R. W. et EHLER, B. D. 1984. a Lethality of Suicide Attempt Rating Scale » Suicide and Life-Threatening Behavior 14 (4) : 215-243.

Strong, M. 1998. A Bright Red Scream: Self-mutilation and the Language of Pain, New York : Viking.

Vasseur, V. 2000. Médecin-chef à la Prison de la Santé, Paris : Cherche-Midi.

4. Martine Herzog-Evans reconnaît d'ailleurs elle-même les limites du droit en ce domaine et, encore plus, le « fantasme totalitaire » à éviter. À preuve, cet articie D $275 \mathrm{du}$ Code de procédure pénal français, qu'elle cite elle-même mais sans insister sur son caractère illusoire. Cet article crée une obligation, non pas à l'administration, mais aux détenus de ne pas " garder à leur disposition tous objets, médicaments ou substances de nature à faciliter notamment le suicide ". Cet accès de bonne volonté de la part du législateur, face à des personnes suicidaires qui n'en ont que faire, nous en fait presque oublier l'interdiction de suicide qui pesait sur tous les citoyens dans les sociétés anciennes. Sur cet aspect historique, voir notamment Robert E. Litman (1980). Voir aussi, pour la situation particulière du Québec, le livre de Guy Grenier (1999). 